\title{
esquema analógico aplicable a secciones de hormigón armado
}

J. NADAL, Dr. Ingeniero de Caminos

\begin{abstract}
Bineopisis
Con émbolos y tubos manométricos se organizan esquemas reológicos de fenómenos resistentes. Se describe el método y se aplica a modelos de secciones de hormigón armado, sometidos a flexión pura. Estos esquemas permiten visualizar el fenómeno desde su fase inicial a la de rotura.
\end{abstract}

A efectos docentes pueden establecerse esquemas analógicos de fenómenos resistentes, sirviéndose de un aparato que, en esencia, se compone de un cilindro con su émbolo, unido a un tubo ascendente. A este tubo, que puede ser de vidrio o de un plástico transparente, se le da forma adecuada para que la fuerza apiicada sobre el émbolo y el recorrido de éste vengan ligados por una función determinada.

En la figura 1 se representa un esquema del aparato, que consta de un cilindro vertical con su émbolo y de un tubo abierto por un extremo y unido por el otro al fondo del cilindro.

La cara superior del émbolo está en comunicación con la atmósfera. La parte inferior del tubo y la del cilindro están llenas de un líquido que en el cilindro llega hasta la cara inferior del émbolo. Si se aplica al émbolo una fuerza hacia abajo, el líquido pasa del cilindro al tubo y el émbolo desciende hasta alcanzar una posición de equilibrio. El problema consiste en disponer el tubo de tal modo que las presiones aplicadas al líquido mediante el émbolo y los recorridos de éste estén ligados por una ley dada.

El tubo puede ser de diámetro constante o variable.

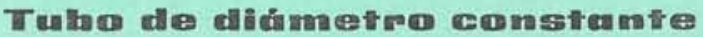

El diámetro del tubo es mucho menor que el del cilindro, y en los modelos que hemos construido, este diámetro es muy pequeño. De aquí que se presente una diferencia entre los niveles del líquido en el tubo y en el cilindro, una vez desprovisto éste del émbolo, según se indica en la figura 2. Esta diferencia de niveles se designa por $p$, considerándose positivos los valores de $p$ cuando el nivel en el cilindro sea el superior, como ocurre con el mercurio.
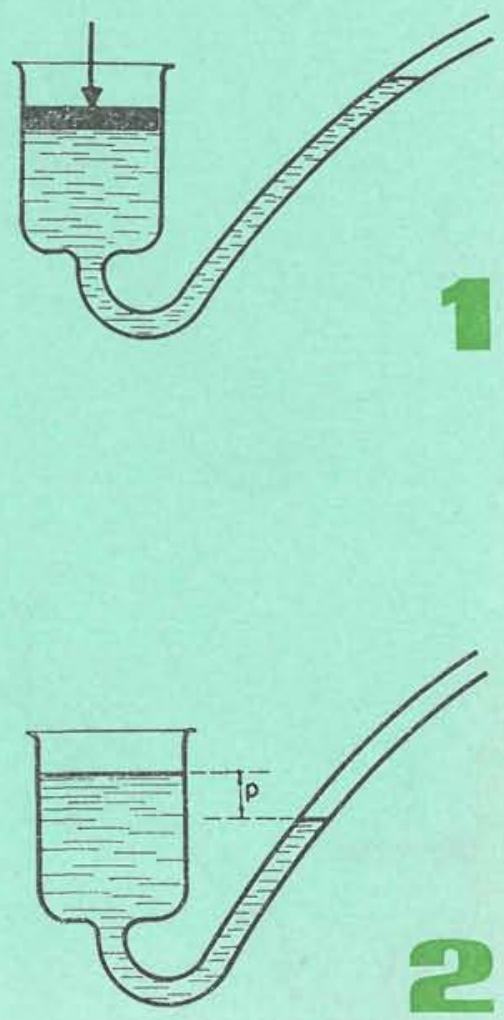

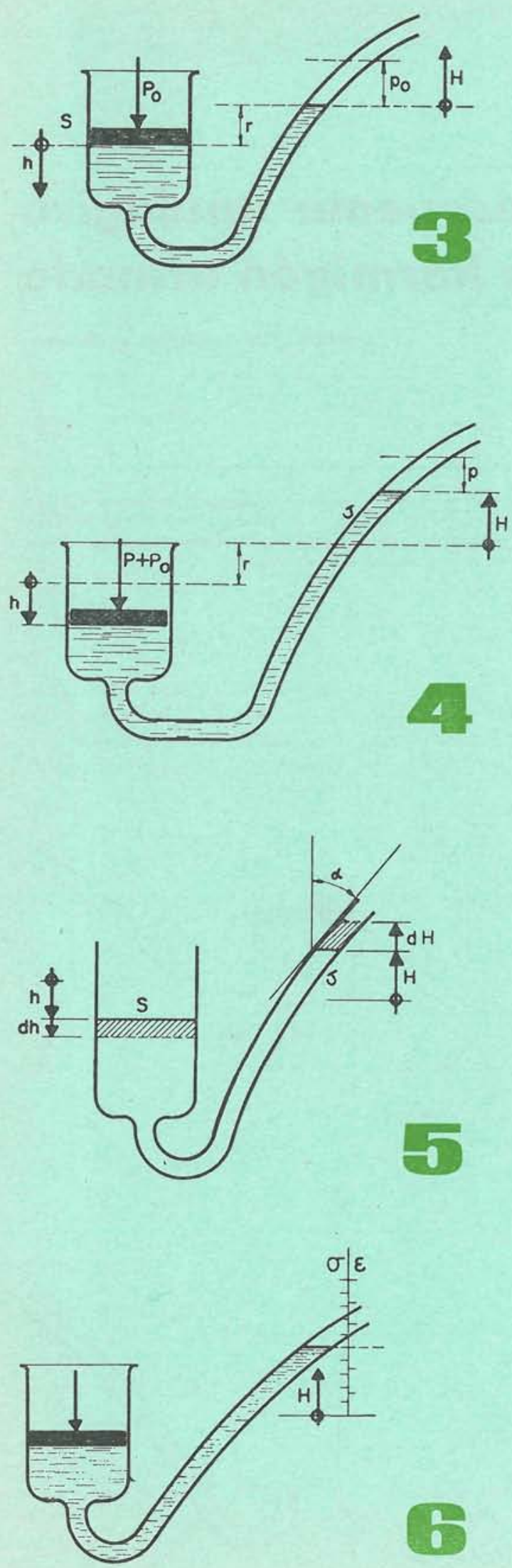

Apliquemos al émbolo una fuerza $P$. La presión ejercida por el émbolo sobre el líquido será la debida a $F=P+P_{o}$, siendo $P_{0}$ el peso del émbolo y el de cualquier dispositivo que se emplee para cargarlo. Con objeto de no tener en cuenta más que las presiones y los recorridos debidos a $P$, que son los que interesa ligar, se procede al tarado del aparato. Para ello (fig. 3 ) se hace $P=o$, con lo que $F=P_{0}$. Debido a $P_{o}$, el líquido alcanzará unos ciertos niveles en el cilindro y en el tubo. Sea $r$ la diferencia entre estos niveles considerada como positiva cuando el nivel en el tubo sea el superior.

Dichos niveles son los orígenes a partir de los cuales se van a medir sus variaciones: $h$ en el cilindro, $H$ en el tubo. Los valores de $h$ se consideran positivos hacia abajo y los de $H$ hacia arriba.

Designemos por $p_{o}$ el correspondiente valor de $p$, por $\delta$ el peso específico del líquido, y por $\mathrm{S}$ la sección del cilindro. Se tiene:

$$
P_{o}=\delta \cdot \mathrm{S}\left(r+p_{o}\right)
$$

Apliquemos una fuerza $P$ al émbolo, que transmitirá al líquido una fuerza $P+P_{o}$ (fig. 4). Se tiene:

$$
P+P_{o}=\delta \cdot \mathrm{S}(r+h+H+p)
$$

Restando de esta expresión la [1], resulta:

$$
P=\delta \cdot S\left(h+H+p-p_{o}\right)
$$

Por otra parte, como el líquido que sale del cilindro es el que entra en el tubo (fig. 5):

$$
S d h=\frac{s}{\cos \alpha} d H
$$

siendo $s$ la sección del tubo y $\alpha$ el ángulo que su eje forma con la vertical en el punto considerado.

El recorrido $h$ del émbolo puede escribirse, por ejemplo:

$$
h=\frac{1.000}{K} \varepsilon
$$

siendo $K$ una constante arbitraria cuya dimensión es la inversa de una longitud y $\varepsilon$ una nueva variable adimensional que puede identificarse con una deformación, expresada en milésimas.

Análogamente podemos escribir:

$$
P=K^{\prime} \sigma
$$


en la cual $K^{\prime}$ es una constante arbitraria cuya dimensión es la de una superficie y $\sigma$ una nueva variable cuya dimensión es la de una tensión, con la cual la identificamos. Con este cambio de variable, la expresión [3] se transforma en:

$$
\sigma=\frac{\delta \cdot S}{K^{\prime}}\left(h+H+p-p_{o}\right)
$$

De [6] se obtiene:

$$
d_{\sigma}=\frac{\delta \cdot S}{K^{\prime}}(d h+d H+d p)
$$

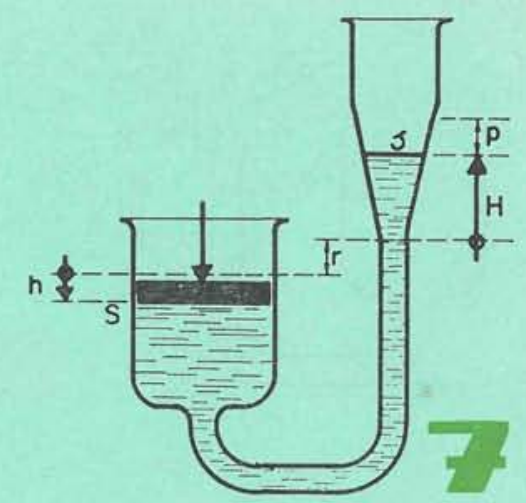

$y$ de $[5]:$

$$
d \varepsilon=\frac{K}{1.000} d h
$$

Designando por $E$ el módulo tangente de elasticidad, se tiene la expresión:

$$
E=\frac{d_{\sigma}}{d_{\varepsilon}}=1.000 \cdot \frac{\delta \cdot \mathrm{S}}{K K^{\prime}}\left(1+\frac{d H}{d h}+\frac{d p}{d h}\right)
$$

que, en virtud de [4], se transforma en la que sigue:

$$
\mathrm{E}=1.000 \cdot \frac{\delta \cdot \mathrm{S}}{K K^{\prime}}\left(1+\frac{\mathrm{S}}{s} \cos \alpha+\frac{d p}{d h}\right)
$$

la cual liga el módulo tangente de elasticidad con el ángulo $\alpha$. Mientras este ángulo sea constante, $E$ también lo será, pues $p$ será constante $\mathrm{y}$, por tanto:

$$
\frac{d p}{d h}=0
$$

En el caso general, dada una ley tensiones-deformaciones $\sigma=\Phi(\varepsilon)$, las expresiones [4], [5] y [6] permiten hallar la ley $\alpha=\alpha(H)$, así como las $\varepsilon=\varepsilon(H)$ y $\sigma=\sigma(H)$. Una doble escala vertical, acotada en $\sigma$ y $\varepsilon$, cuyo origen es común y coincidente con el de $H$, permite asociar a cada nivel del líquido en el tubo, es decir, a cada valor de $H$, tanto el valor de $\sigma$ como el de $\varepsilon$ correspondiente, como se indica en la figura 6. Los recorridos $h \mathrm{y}$, por lo tanto, las deformaciones $\varepsilon$, son proporcionales a las longitudes, medidas a partir del origen de $H$, de la parte del tubo ocupada por el líquido.

Los tubos de sección constante tienen la ventaja de reproducir la curva tensión-deformación en forma en cierto modo análoga a la que aparece en los registradores de las máquinas de ensayo y en los libros. Tienen, en cambio, el inconveniente de que las ramas curvas son muy largas para materiales con tramos de fluencia dilatados, como son los aceros ordinarios. Por ello resulta más práctico variar el diámetro del tubo y mantener éste vertical (fig. 7), con lo que ocupa menos espacio.

Las expresiones obtenidas para $s$ constante siguen siendo válidas sin más que hacer en ellas $\cos \alpha=1$ y considerar $s$ variable. Las expresiones [4], [5] y [6] quedan:

$$
\mathrm{S} d h=s d H \quad ; \quad h=\frac{1.000}{K} \varepsilon \quad ; \quad \sigma=\frac{\delta \cdot \mathrm{S}}{K^{\prime}}\left(h+H+p-p_{o}\right)
$$



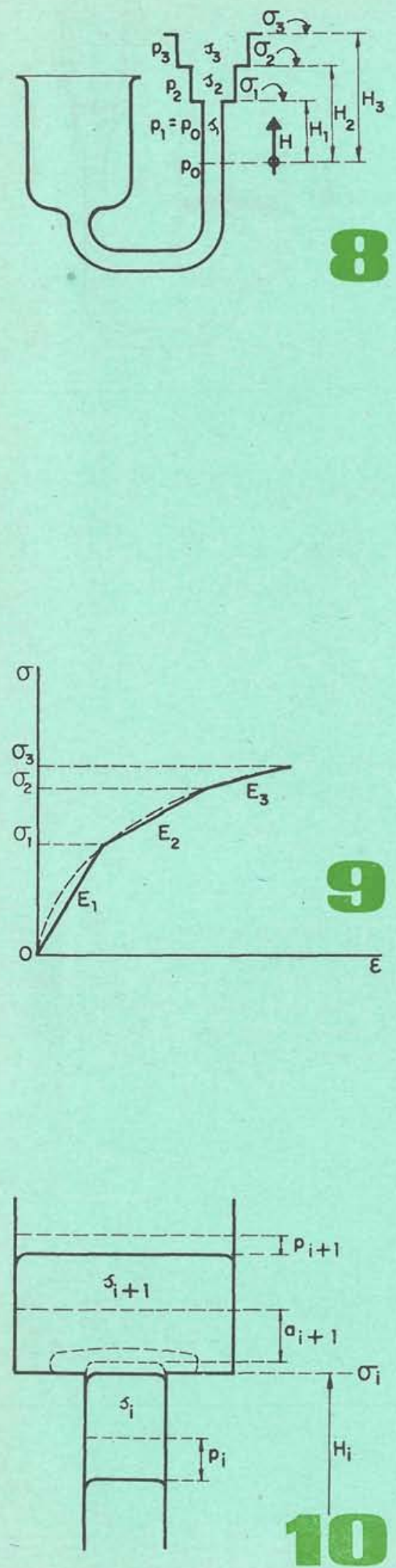

Estas expresiones, junto con la ley $\sigma=\Phi(\varepsilon)$, permiten obtener la $s=s(H)$ y, posteriormente, las $\varepsilon=\varepsilon(H)$ y $\sigma=\sigma(H)$.

La dificultad práctica de realizar un tubo cuya sección varíe de modo impuesto aconseja sustituirlo por trozos de sección constante (fig. 8), pero variable de un trozo a otro. Esto equivale, como vamos a ver a continuación, a sustituir el diagrama dado tensiones-deformaciones, por otro (fig. 9) formado por tramos rectos en cada uno de los cuales el módulo $E$ será constante.

La expresión [7] aplicada a tubos verticales de sección variable queda:

$$
E=1.000 \frac{\delta \cdot S}{K K^{\prime}}\left(1+\frac{S}{s}+\frac{d p}{d h}\right)
$$

Para cada trozo de tubo, cuya sección no varía, será $d p / d h=0$, con lo que se tendrá:

$$
E=1.000 \frac{\delta \cdot S}{K K^{\prime}}\left(1+\frac{S}{s}\right)
$$

Como vemos, al ser $s$ constante, $E$ también lo es. Por tanto cada trozo del tubo, por ser de $s$ constante, corresponde a un valor de $E$.

Una vez fijados los valores de $\delta, S, K$ y $K^{\prime}$, como no disponemos más que de unos ciertos valores de $s$ no dispondremos más que de otros tantos valores de $E$, a los cuales habrá que acomodarse para sustituir el diagrama tensiones-deformaciones por el formado de trozos rectos.

Para definir el tubo sólo falta determinar las longitudes de sus trozos. De [8] se deduce inmediatamente que, para cada trozo, $h$ varía linealmente con $H \mathrm{y}$, por tanto, $\sigma$ varía linealmente con $H$, dependiendo esta variación del trozo de tubo que se considere.

Ahora bien, si el líquido es mercurio y el tubo es de vidrio, el menisco tiene su concavidad hacia abajo. Por tanto (fig. 10) si, como suponemos, no consideramos más valores de $p$ que los correspondientes a las secciones de los diversos trozos del tubo, las fórmulas anteriores perderán su validez cuando el nivel del líquido esté dentro de una pequeña zona situada en las proximidades de los cambios de sección y por encima de estos cambios. La altura de dicha zona, $a_{i+1}$ en la figura 10 , variará con la sección del trozo.

El valor $\sigma_{i}$ de $\sigma$, asociado a un cambio de sección en el que $H=H_{i}$, será el correspondiente a este valor de $H$ para el tubo inferior.

Designemos por $h_{1}, h_{2}, \ldots$, los valores de $h$ correspondientes a los $H_{1}, H_{2}, \ldots$, de $H$, en los que se verifican los cambios de sección. 
De acuerdo con $[8]$ se tiene:

$$
\begin{gathered}
\sigma_{1}=\frac{\delta \cdot S}{K^{\prime}}\left(h_{1}+H_{1}+p_{1}-p_{o}\right) ; p_{1}=p_{o} \\
\sigma_{2}=\frac{\delta \cdot S}{K^{\prime}}\left(h_{2}+H_{2}+p_{2}-p_{o}\right) \\
\sigma_{3}=\frac{\delta \cdot S}{K^{\prime}}\left(h_{3}+H_{3}+p_{3}-p_{o}\right)
\end{gathered}
$$

Por otra parte:

$$
\begin{gathered}
\mathrm{S} h_{1}=s_{1} H_{1} \\
\mathrm{~S}\left(h_{2}-h_{1}\right)=s_{2}\left(H_{2}-H_{1}\right) \\
\mathrm{S}\left(h_{3}-h_{2}\right)=s_{3}\left(H_{3}-H_{2}\right)
\end{gathered}
$$

Por tanto:

$$
\begin{gathered}
H_{1}=\sigma_{1} \frac{K^{\prime}}{\delta\left(\mathrm{S}+s_{1}\right)} \\
H_{2}-H_{1}=\left(\sigma_{2}-\sigma_{1}\right) \frac{\boldsymbol{K}^{\prime}}{\delta\left(\mathrm{S}+s_{2}\right)}- \\
-\frac{\mathrm{S}}{\mathrm{S}+s_{2}}\left(p_{2}-p_{1}\right) \\
H_{3}-H_{2}=\left(\sigma_{3}-\sigma_{2}\right) \frac{K^{\prime}}{\delta\left(\mathrm{S}+s_{3}\right)}- \\
-\frac{\mathrm{S}}{\mathrm{S}+s_{3}}\left(p_{3}-p_{2}\right)
\end{gathered}
$$

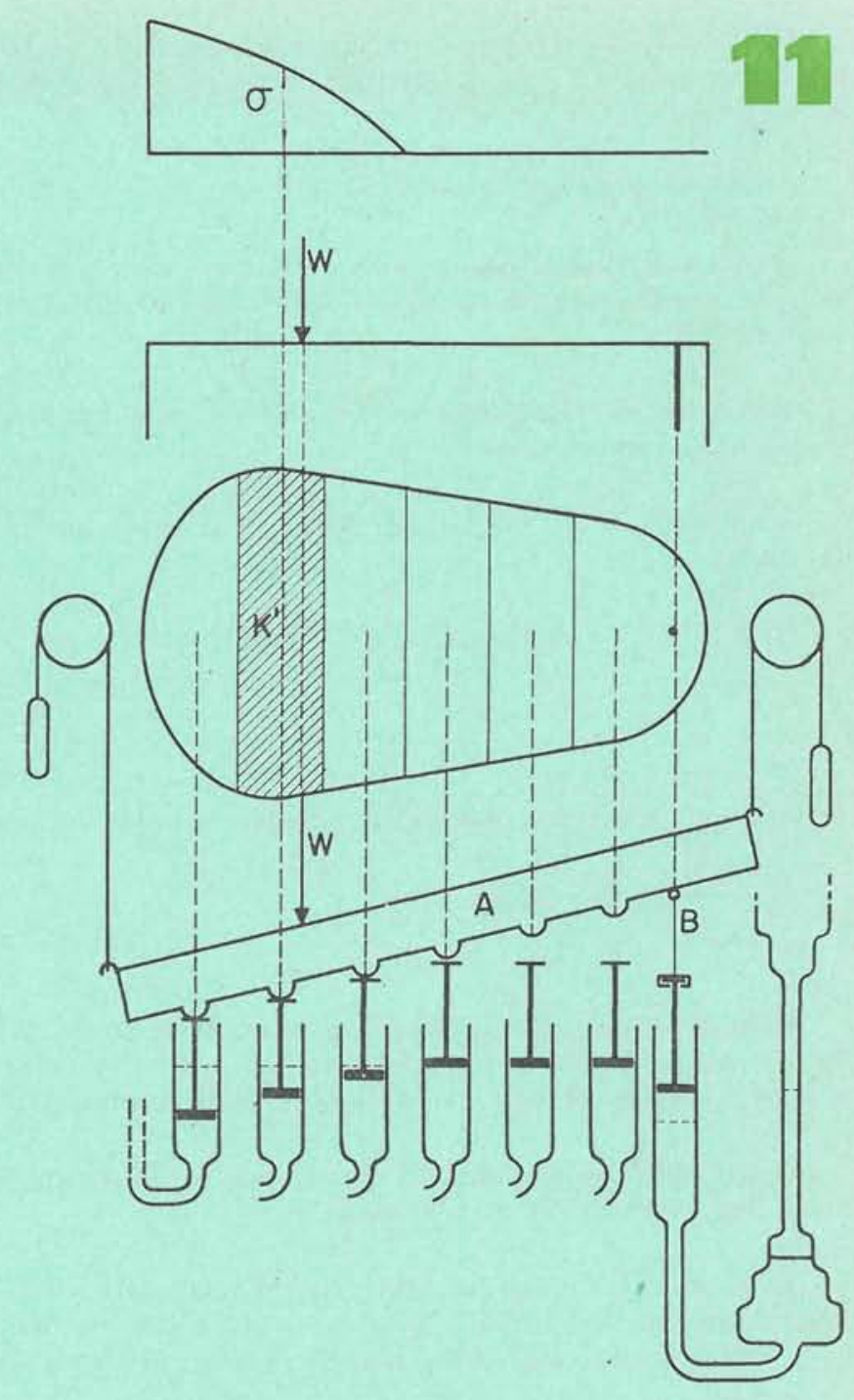

Estas expresiones permiten hallar los valores de $H_{1}, H_{2}, H_{3}, \ldots$ (fig. 8), una vez conocidos los de $\sigma_{1}, \sigma_{2}, \sigma_{3}, \ldots$ (fig. 9), correspondientes a los vértices del diagrama formado por trozos rectos, que sustituye al real de tensiones-deformaciones.

Una vez definido el tubo, para hallar el valor de $\sigma$ asociado a cualquier valor de $H$, basta aplicar la tercera de las fórmulas [8], una vez hallado el valor de $h$ correspondiente al de $H$, lo que es inmediato, pues para ello basta expresar que el volumen $S h$ es igual que el del tubo, desde $H=0$ hasta el de $H$ en cuestión.

Esto es válido, salvo para las pequeñas zonas próximas a los cambios de sección, y a las cuales nos hemos referido anteriormente, donde puede establecerse una variación gradual de $\sigma$.

\section{Aplicación al horamiggón carmeato}

Consideremos el aparato de la figura 11 compuesto, fundamentalmente, por una barra, $A$, y varias unidades como la descrita anteriormente, cada una de las cuales consta de un émbolo, un cilindro $y$ un tubo. 
Cada unidad corresponde a un diagrama dado de tensiones-deformaciones y, por tanto, a un material. En la figura se aprecian seis tubos correspondientes al hormigón y uno correspondiente a una armadura.

Al cargar mediante la fuerza $W$ la barra $A$, ésta acciona los émbolos.

Suponemos que el hormigón no trabaja a tracción. Por tanto, el aparato está dispuesto para que la barra $A$ pueda empujar hacia abajo los émbolos correspondientes a este material, pero no tirar de ellos hacia arriba.

En cuanto al acero, la barra $A$ puede empujar hacia abajo y tirar hacia arriba el émbolo correspondiente a la armadura.

Admitimos la ley de Navier, según la cual las secciones inicialmente planas siguen siéndolo al deformarse.

La barra $A$ materializa esta ley, por lo que debe ser recta y rígida. Puesto que es

$$
h=\frac{1.000}{K} \varepsilon
$$

la constante arbitraria $K$ debe ser común a todas las unidades.

Por otra parte se tiene:

$$
P=\sigma K^{\prime}
$$

Identifiquemos la tensión $\sigma$ con la experimentada por el material correspondiente a la unidad de que se trate. Podemos, pues, asociar esta unidad a una superficie $K^{\prime}$ de dicho material. Naturalmente, $\sigma$ será la tensión de este material en el punto correspondiente al émbolo.

Claro está que si todas las superficies $K^{\prime}$ del hormigón son iguales, todos los tubos correspondientes a este material también lo serán.

El aparato sin carga, es decir, con $W=0$, debe tener la barra $A$ en contacto con los émbolos correspondientes al hormigón y el niveı del lıquiao en el origen de las $H$ en todos los tubos. No es necesario que los niveles del líquido en los cilindros estén en un mismo plano horizontal.

Para que estas condiciones se cumplan, se vierte más o menos líquido en cada unidad, de modo que ésta, una vez provista de su émbolo, cumpla la condición referente al nivel del líquido en el tubo, y luego se desplaza la unidad completa hasta que ocupe la posición adecuada.

Finalmente, y con objeto de descartar el peso de la barra $A$ y el de la $B$, unida a aquélla y al émbolo de la unidad correspondiente a la armarura. se proveen dos contrapesos con objeto de que el conjunto de las barras $A$ y $B$ estén en equilibrio indiferente.

\section{Estudio amentitico}

Seguidamente vamos a estudiar la traducción analítica del comportamiento del aparato aplicado a una sección rectangular de hormigón armado sin armadura de compresión, sometida a flexión pura.

Designemos por $\varepsilon_{b}^{\prime}$ y $\varepsilon_{a}^{\prime}$ (fig. 12) las deformaciones del hormigón en el borde más comprimido y de la armadura, respectivamente.

Siendo $x$ la profundidad de la línea neutra, y $h$ el canto útil, se tiene:

$$
\frac{x}{h}=\frac{\varepsilon_{b}^{\prime}}{\varepsilon_{a}^{\prime}+\varepsilon_{b}^{\prime}}
$$


De acuerdo con $[8]$ se tiene:

$$
\begin{gathered}
\sigma_{1}=\frac{\delta \cdot S}{K^{\prime}}\left(h_{1}+H_{1}+p_{1}-p_{o}\right) ; p_{1}=p_{o} \\
\sigma_{2}=\frac{\delta \cdot S}{K^{\prime}}\left(h_{2}+H_{2}+p_{2}-p_{o}\right) \\
\sigma_{3}=\frac{\delta \cdot S}{K^{\prime}}\left(h_{3}+H_{3}+p_{3}-p_{o}\right)
\end{gathered}
$$

Por otra parte:

$$
\begin{gathered}
\mathrm{S} h_{1}=s_{1} H_{1} \\
\mathrm{~S}\left(h_{2}-h_{1}\right)=s_{2}\left(H_{2}-H_{1}\right) \\
\mathrm{S}\left(h_{3}-h_{2}\right)=s_{3}\left(H_{3}-H_{2}\right)
\end{gathered}
$$

Por tanto:

$$
\begin{gathered}
H_{1}=\sigma_{1} \frac{K^{\prime}}{\delta\left(\mathrm{S}+s_{1}\right)} \\
H_{2}-H_{1}=\left(\sigma_{2}-\sigma_{1}\right) \frac{K^{\prime}}{\delta\left(\mathrm{S}+s_{2}\right)}- \\
-\frac{\mathrm{S}}{\mathrm{S}+s_{2}}\left(p_{2}-p_{1}\right) \\
H_{3}-H_{2}=\left(\sigma_{3}-\sigma_{2}\right) \frac{K^{\prime}}{\delta\left(\mathrm{S}+s_{3}\right)}- \\
-\frac{\mathrm{S}}{\mathrm{S}+s_{3}}\left(p_{3}-p_{2}\right)
\end{gathered}
$$

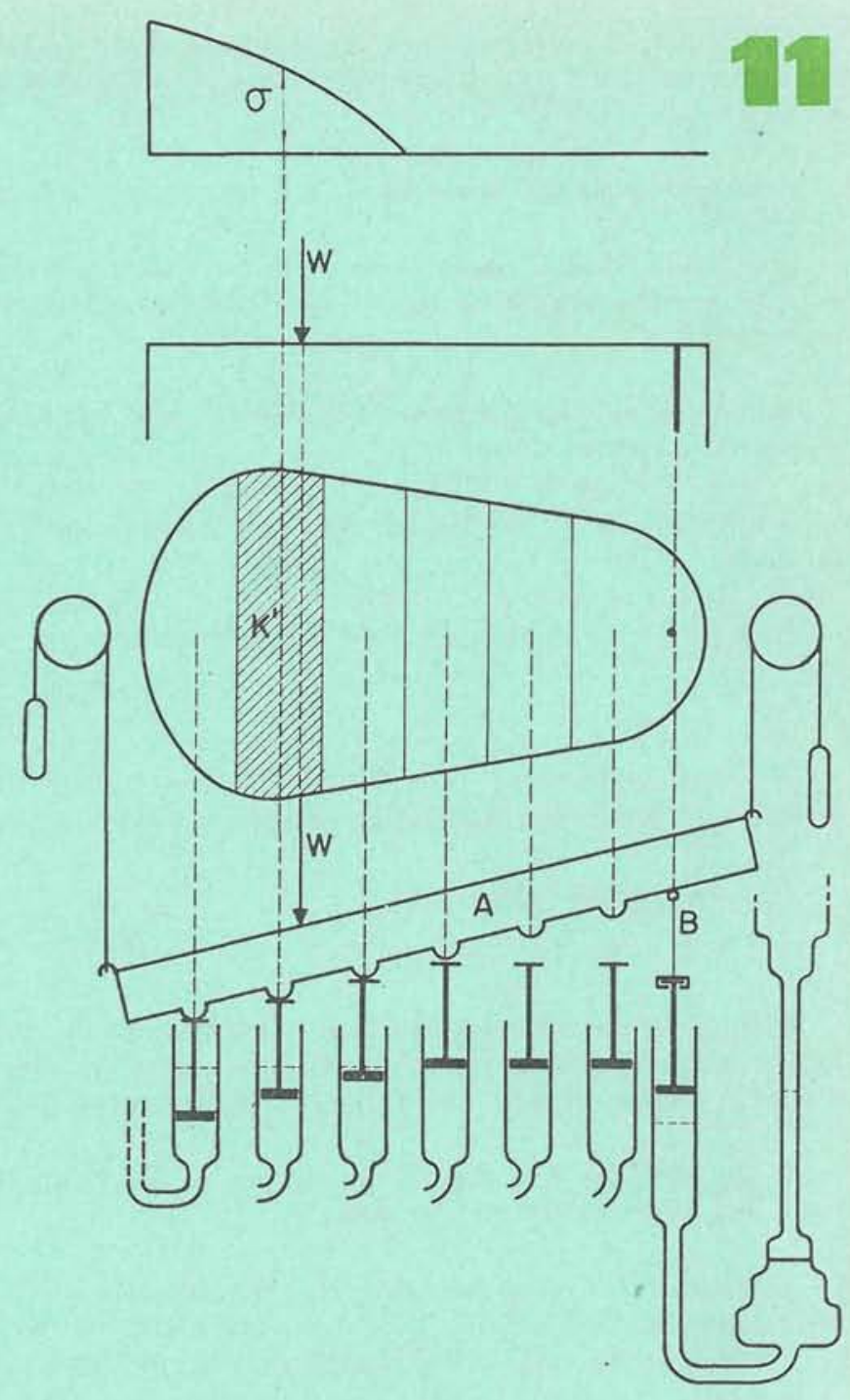

Estas expresiones permiten hallar los valores de $H_{1}, H_{2}, H_{3}, \ldots$ (fig. 8), una vez conocidos los de $\sigma_{1}, \sigma_{2}, \sigma_{3}, \ldots$ (fig. 9), correspondientes a los vértices del diagrama formado por trozos rectos, que sustituye al real de tensiones-deformaciones.

Una vez definido el tubo, para hallar el valor de $\sigma$ asociado a cualquier valor de $H$, basta aplicar la tercera de las fórmulas [8], una vez hallado el valor de $h$ correspondiente al de $H$, lo que es inmediato, pues para ello basta expresar que el volumen $\mathrm{S} h$ es igual que el del tubo, desde $H=0$ hasta el de $H$ en cuestión.

Esto es válido, salvo para las pequeñas zonas próximas a los cambios de sección, y a las cuales nos hemos referido anteriormente, donde puede establecerse una variación gradual de $\sigma$.

\section{Aplíceceión call homanigón carmeado}

Consideremos el aparato de la figura 11 compuesto, fundamentalmente, por una barra, A, y varias unidades como la descrita anteriormente, cada una de las cuales consta de un émbolo, un cilindro $y$ un tubo. 
Cada unidad corresponde a un diagrama dado de tensiones-deformaciones $\mathrm{y}$, por tanto, a un material. En la figura se aprecian seis tubos correspondientes al hormigón y uno correspondiente a una armadura.

$\mathrm{Al}$ cargar mediante la fuerza $W$ la barra $A$, ésta acciona los émbolos.

Suponemos que el hormigón no trabaja a tracción. Por tanto, el aparato está dispuesto para que la barra $A$ pueda empujar hacia abajo los émbolos correspondientes a este material, pero no tirar de ellos hacia arriba.

En cuanto al acero, la barra $A$ puede empujar hacia abajo y tirar hacia arriba el émbolo correspondiente a la armadura.

Admitimos la ley de Navier, según la cual las secciones inicialmente planas siguen siéndolo al deformarse.

La barra $A$ materializa esta ley, por lo que debe ser recta y rígida. Puesto que es

$$
h=\frac{1.000}{K} \varepsilon
$$

la constante arbitraria $K$ debe ser común a todas las unidades.

Por otra parte se tiene:

$$
P=\sigma K^{\prime}
$$

Identifiquemos la tensión $\sigma$ con la experimentada por el material correspondiente a la unidad de que se trate. Podemos, pues, asociar esta unidad a una superficie $K^{\prime}$ de dicho material. Naturalmente, $\sigma$ será la tensión de este material en el punto correspondiente al émbolo.

Claro está que si todas las superficies $K^{\prime}$ del hormigón son iguales, todos los tubos correspondientes a este material también lo serán.

El aparato sin carga, es decir, con $W=0$, debe tener la barra $A$ en contacto con los émbolos correspondientes al hormigón y el niveı del lıquiao en el origen de las $H$ en todos los tubos. No es necesario que los niveles del líquido en los cilindros estén en un mismo plano horizontal.

Para que estas condiciones se cumplan, se vierte más o menos líquido en cada unidad, de modo que ésta, una vez provista de su émbolo, cumpla la condición referente al nivel del líquido en el tubo, y luego se desplaza la unidad completa hasta que ocupe la posición adecuada.

Finalmente, y con objeto de descartar el peso de la barra $A$ y el de la $B$, unida a aquélla y al émbolo de la unidad correspondiente a la armarura. se proveen dos contrapesos con objeto de que el conjunto de las barras $A$ y $B$ estén en equilibrio indiferente.

\section{Estudio anculitico}

Seguidamente vamos a estudiar la traducción analítica del comportamiento del aparato aplicado a una sección rectangular de hormigón armado sin armadura de compresión, sometida a flexión pura.

Designemos por $\varepsilon_{b}^{\prime} \mathrm{y}^{\prime} \varepsilon_{a}$ (fig. 12) las deformaciones del hormigón en el borde más comprimido y de la armadura, respectivamente.

Siendo $x$ la profundidad de la línea neutra, y $h$ el canto útil, se tiene:

$$
\frac{x}{h}=\frac{\varepsilon_{b}^{\prime}}{\varepsilon_{a}^{\prime}+\varepsilon_{b}^{\prime}}
$$



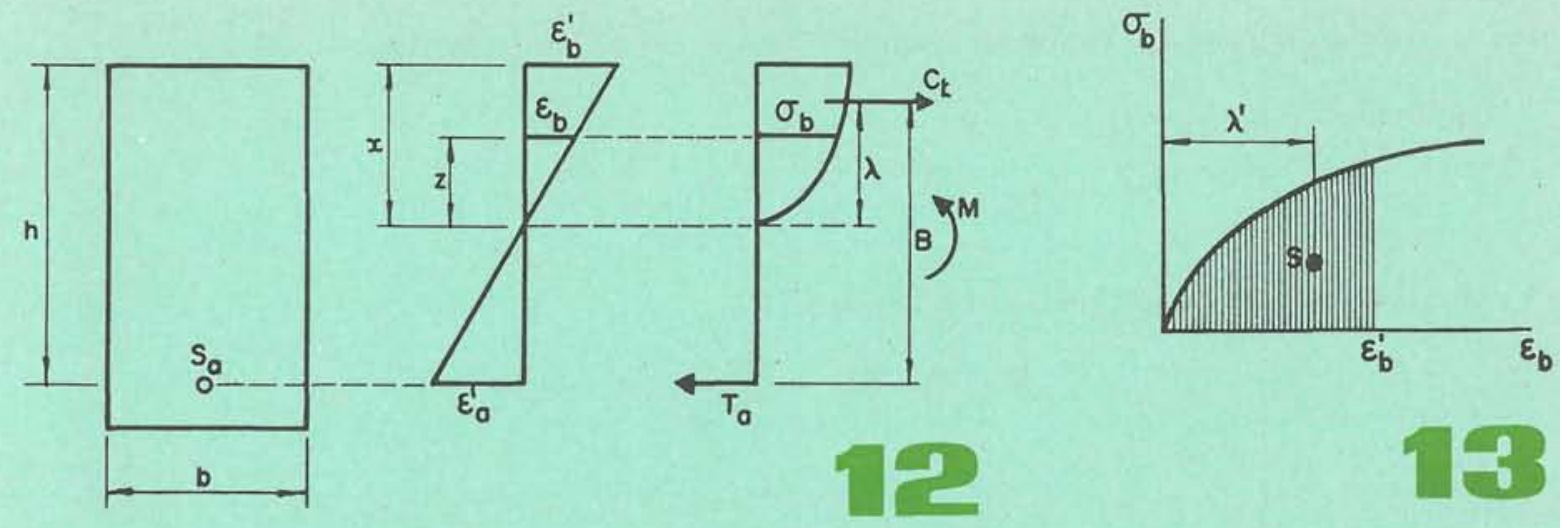

La deformación $\varepsilon_{b}$ del hormigón comprimido a una distancia $z$ de la línea neutra será:

$$
\varepsilon_{b}=\varepsilon_{b}^{\prime} \frac{z}{x}
$$

La resultante $C_{b}$ de las compresiones en el hormigón es:

$$
C_{b}=b \int_{0}^{x} \sigma_{b} d z=\frac{b h}{\varepsilon_{a}^{\prime}+\varepsilon_{b}^{\prime}} \int_{0}^{\varepsilon_{b}^{\prime}} \sigma_{d} \varepsilon_{b}=\frac{b h}{\varepsilon_{a}^{\prime}+\varepsilon_{b}^{\prime}} \cdot S
$$

donde $b$ es el ancho de la sección y $\sigma_{b}$ la compresión del hormigón para la deformación $\varepsilon_{b}$. La relación que vincula $\sigma_{b}$ y $\varepsilon_{b}$ es la dada por el diagrama de tensiones-deformaciones para dicho material. Por tanto, $\mathrm{S}$ es el área rayada en la figura 13.

La fuerza de tracción $T_{a}$ de la armadura será:

$$
T_{a}=\mathrm{S}_{a} \cdot \sigma_{a}^{\prime}
$$

en la que $S_{a}$ es la sección de la armadura y $\sigma^{\prime}{ }_{a}$ la tensión del acero que el diagrama tensiones-deformaciones del acero da para la deformación $\varepsilon_{a}^{\prime}$.

La igualdad de tracciones y compresiones da:

$$
C_{b}=T_{a}
$$

y, por tanto:

$$
q=\frac{S_{a}}{b h}=\frac{S}{\left(\varepsilon_{a}^{\prime}+\varepsilon_{b}^{\prime}\right) \sigma_{a}^{\prime}}
$$

La distancia $\lambda$ de $C_{b}$ a la línea neutra viene dada por:

$$
\lambda=\frac{b \int_{0}^{\mathrm{x}} \sigma_{b} z d z}{C_{b}}=\frac{\frac{b h^{2}}{\left(\varepsilon_{a}^{\prime}+\varepsilon_{b}^{\prime}\right)^{2}} \int_{0}^{\varepsilon_{b}^{\prime}} \sigma_{b} \varepsilon_{b} d \varepsilon_{b}}{C_{b}}
$$

o sea :

$$
\lambda=\frac{h}{\varepsilon_{a}^{\prime}+\varepsilon_{b}^{\prime}} \cdot \frac{\int_{0}^{\varepsilon_{b}^{\prime}} \sigma_{b} \varepsilon_{b} d \varepsilon_{b}}{S}=\frac{h}{\varepsilon_{a}^{\prime}+\varepsilon_{b}^{\prime}} \cdot \lambda^{\prime}
$$


donde $\lambda^{\prime}$ es la distancia del centro de gravedad de $S$ al eje de las $\sigma_{b}$ (fig. 13).

La distancia $B$ de $C_{b}$ a $S_{a}$ es:

$$
B=\lambda+h-x=\frac{h}{\varepsilon_{a}^{\prime}+\varepsilon_{b}^{\prime}}\left(\lambda^{\prime}+\varepsilon_{a}^{\prime}\right)
$$

Por tanto, el momento $M$ que solicita la sección es:

$$
M=C_{b} \cdot B=\frac{b h^{2} S}{\left(\varepsilon_{a}^{\prime}+\varepsilon_{b}^{\prime}\right)^{2}}\left(\lambda^{\prime}+\varepsilon_{a}^{\prime}\right)
$$

de donde:

$$
\frac{M}{b h^{2}}=\frac{S\left(\lambda^{\prime}+\varepsilon_{a}^{\prime}\right)}{\left(\varepsilon_{a}^{\prime}+\varepsilon_{b}^{\prime}\right)^{2}}
$$

Finalmente, el giro $d \theta$ de una cara de la rebanada elemental con respecto a la otra, distante $d s$ de la primera, es:

$$
d \theta=\frac{\left(\varepsilon_{a}^{\prime}+\varepsilon_{b}^{\prime}\right) d s}{\mathrm{~h}}
$$

de donde:

$$
h \frac{d \theta}{d s}=\varepsilon_{a}^{\prime}+\varepsilon_{b}^{\prime}
$$

El gráfico I representa las fórmulas [10], [11], [12] y [13] en una red de coordenadas $\varepsilon_{a}^{\prime}, \varepsilon_{b}^{\prime}$ para los diagramas, tensiones-deformaciones que se indican en él. Como puede apreciarse, se trata de un acero cuyo límite de proporcionalidad es aproximadamente de $2.400 \mathrm{~kg} / \mathrm{cm}^{2}$. El hormigón tiene una carga en rotura de $190 \mathrm{~kg} / \mathrm{cm}^{2}$ y una deformación total de aproximadamente $\varepsilon_{b}=0,5$ por 100 . Aun cuando este alargamiento es superior al normal para las velocidades de carga usuales en los ensayos, hemos creído oportuno incluirlo en el ejemplo para mayor claridad de representación.

En el tercer cuadrante de la figura se representan las curvas correspondientes a la cuantía geométrica $q=\mathrm{S}_{a} / b h$; a la profundidad unitaria de la fibra neutra $x / h$; al momento flector unitario $M / b h^{2}$ para los materiales considerados. Finalmente se representa también el valor de $h d \theta / d s\left(^{*}\right)$.

Este gráfico permite apreciar, por ejemplo, que en el caso de una cuantía $q=2$ por 100 y un momento unitario $M / b h^{2}=50$ para los materiales considerados, el alargamiento $\varepsilon_{b}^{\prime}$ del hormigón es de 0,175 por 100 y la tensión de este material alcanza el valor de $170 \mathrm{~kg} / \mathrm{cm}^{2}$. El acero, por su parte, trabaja a una tensión de $3.080 \mathrm{~kg} / \mathrm{cm}^{2}$ y su alargamiento es de 0,14 por 100 . La profundidad de la fibra neutra (interpolando en las líneas de punto y trazos) es de $x / h=0,54$, y, finalmente, que el giro de una sección respecto de otra a una distancia $h$, igual al canto, es de 0,0032 .

El gráfico III recoge la variación de los estados de tensiones y deformaciones de la sección y materiales considerados en el gráfico I, al crecer el momento flector.

Cada fila de secciones corresponde a la cuantía que se indica, y puede observarse claramente la diferencia de comportamientos en función de la cuantía, en el sentido de variación de la fibra neutra y zona afectada por la plastificación del hormigón.

Los gráficos II señalan la variación de profundidad de fibra neutra y de $h d \theta / d s$, al aumentar cuantías y momentos, todo ello referido, naturalmente, a la sección y materiales considerados en el gráfico anterior.

$\left(^{*}\right) \quad d s=$ diferencial de longitud pieza.

$$
d \theta=\text { diferencial de giro. }
$$




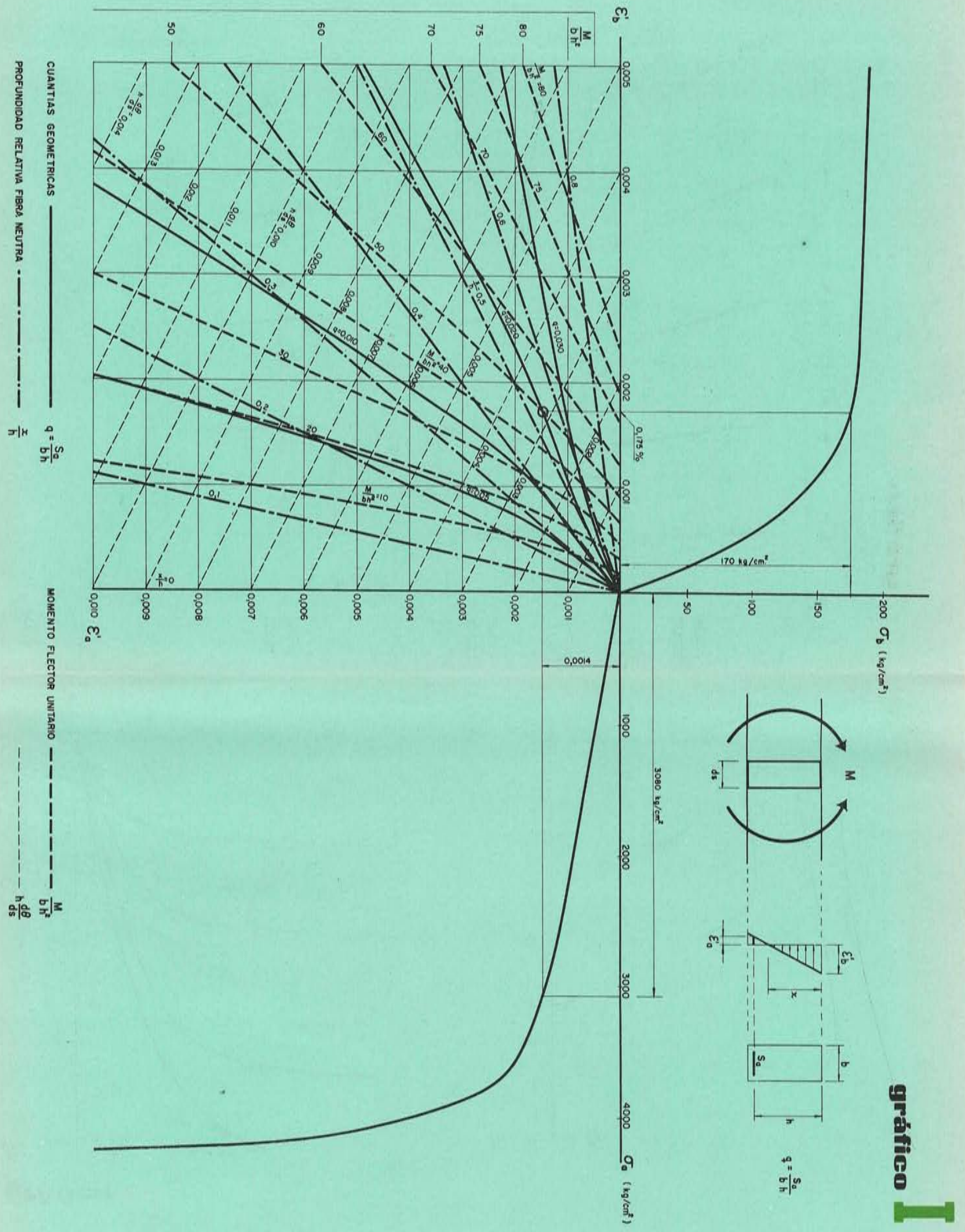



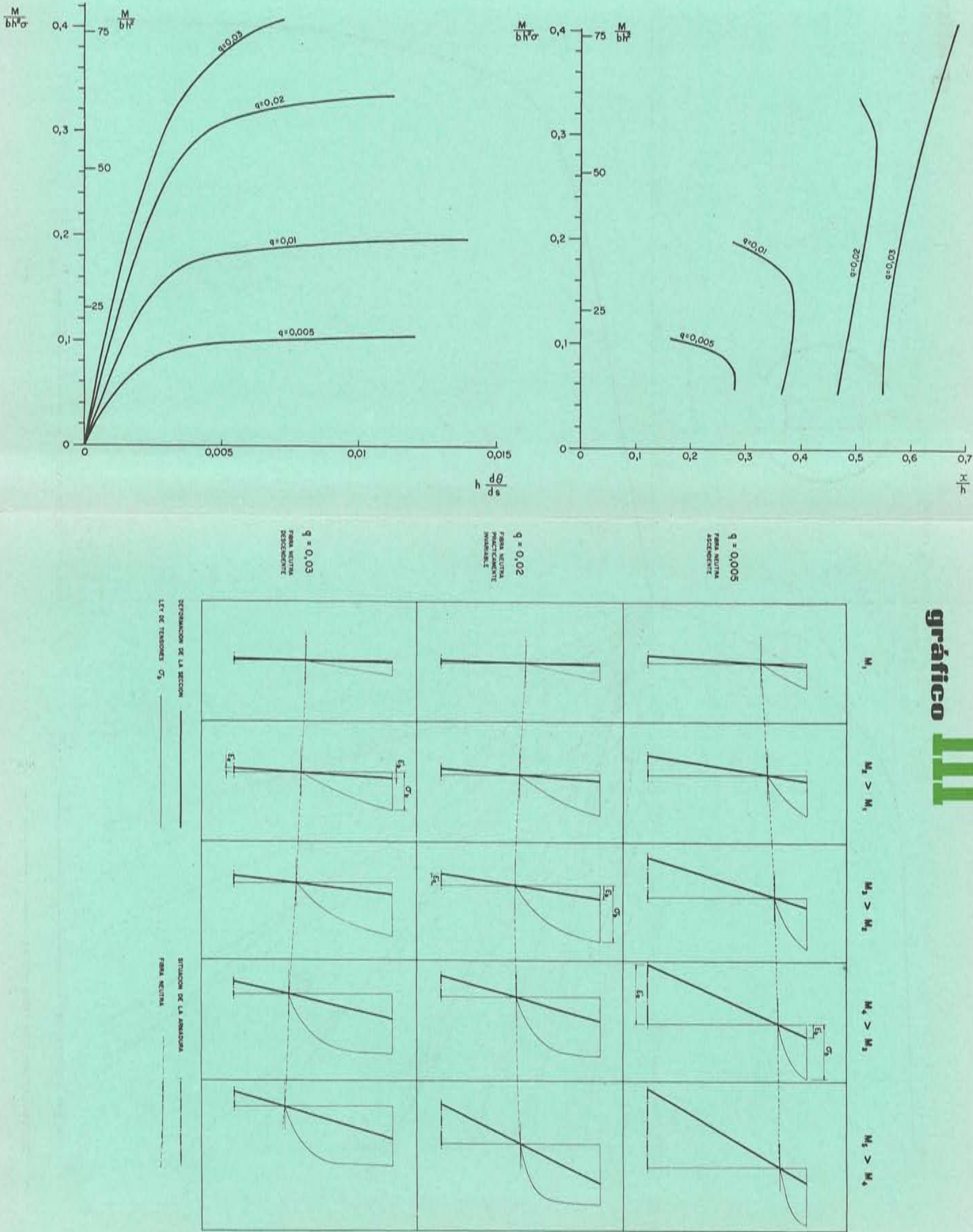


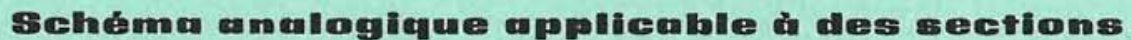

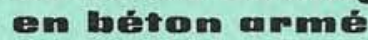

J. Nadal, Dr. ingénieur des Ponts et Chaussées.

A l'aide de pistons et de tubes manométriques, sont organisés des schémas rhéologiques de phénomènes résistants. Cette méthode est décrite et appliquée à des modèles de sections en béton armé, soumis à flexion pure. Ces schémas permettent la visualisation du phénomène de sa phase initiale à sa rupture.

\section{Anculogical pattern applicable to meinforomed concegete sections}

J. Nadal, Dr. Civil Engineer.

Rheological patterns describing strength phenomena have been made with pistons and pressure tubes. The method is described, and the patterns are applied to models of reinforced concrete sections, subjected to pure bending. These patterns make it possible to visualize the phenomena from the initial phase until failure occurs.

\section{Amaloges Selheme anwendiber muf Sitculhubetomequerseghnitto}

Dr. J. Nadal, Ingenieur.

Mit Kolben und Röhrenmanometern werden rheologische Schemen von Widerstandserscheinungen aufgestellt. Es wird die Methode erklärt und an Stahlbetonquerschnitten, die man einer reinen Biegung aussetzt, angewandt.

Die Schemen erlauben eine Boebachtung der Erscheinung von der Anfangs- bis zur Bruchphase. 


\section{untimas publicakiones}

\section{recomendaciones prácticas del Comité Europeo del Hormigón}

(en prensa)

A los diez años de su fundación, el C. E. B. ha ultimado este Reglamento unificado a escala internacional. Se abandonan los métodos clásicos de cálculo del hormigón armado y se da entrada al llamado «cálculo en rotura», aprovechando el mejor conocimiento (obtenido por vía experimental) del comportamiento de los materiales, especialmente en su fase plástica. La teoría semiprobabilista de la seguridad y la noción de los «estados límites son dos de los pilares de este nuevo Reglamento, cuya aplicación conduce a proyectar, calcular y construir con mayor eficacia. Es decir, de un modo más científico, más seguro y más económico.

\section{del \\ hormigón armado \\ cálloula Bn rofura} (a)

\section{instrucción}

h. a. 61

\section{del Instituto \\ Eduardo Torroja}

De todas las existentes, esta Instrucción es la primera, y única hasta la fecha, que se ajusta en sus líneas generales a las «Recomendaciones» del C. E. B. Publicada hace más de dos años, introduce el concepto de «resistencia característica», desarrolla la teoría semiprobabilista de la seguridad y pone a punto el cálculo en rotura por el método del «momento tope», una de las más valiosas contribuciones de su creador, Eduardo Torroja, al cálculo del hormigón armado. El técnico familiarizado con esta Instrucción encontrará fácil el acceso a las «Recomendaciones del C. E. B., y sacará de ellas el mayor provecho.

\section{materiales \\ ejecución}

documentos del proyecto cálculo de piezas lineales planas tomo I

tomo II (interpretación estadística)
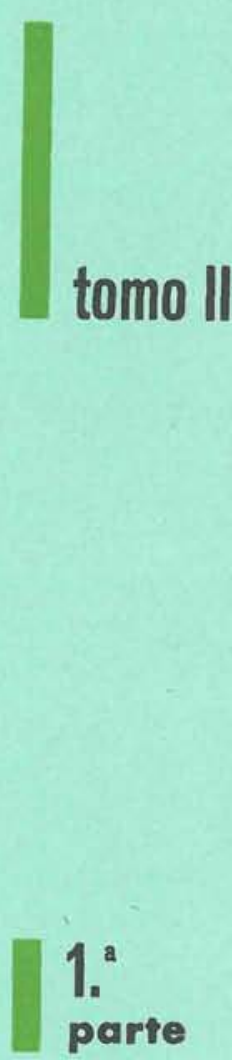

2." y 3. parte 


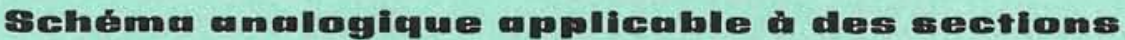 Can bécợ cummé}

J. Nadal, Dr. ingénieur des Ponts et Chaussées.

A l'aide de pistons et de tubes manométriques, sont organisés des schémas rhéologiques de phénomènes résistants. Cette méthode est décrite et appliquée à des modèles de sections en béton armé, soumis à flexion pure. Ces schémas permettent la visualisation du phénomène de sa phase initiale à sa rupture.

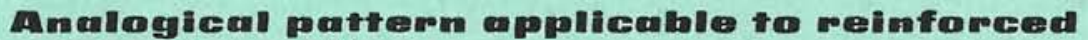 concerete sections}

J. Nadal, Dr. Civil Engineer.

Rheological patterns describing strength phenomena have been made with pistons and pressure tubes. The method is described, and the patterns are applied to models of reinforced concrete sections, subjected to pure bending. These patterns make it possible to visualize the phenomena from the initial phase until failure occurs.

\section{Amaloges Schemes arewemdibar auf

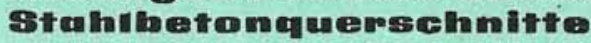

Dr. J. Nadal, Ingenieur.

Mit Kolben und Röhrenmanometern werden rheologische Schemen von Widerstandserscheinungen aufgestellt. Es wird die Methode erklärt und an Stahlbetonquerschnitten, die man einer reinen Biegung aussetzt, angewandt.

Die Schemen erlauben eine Boebachtung der Erscheinung von der Anfangs- bis zur Bruchphase. 